\title{
Current states in an SNS junction for arbitrary thickness of the normal layer at temperatures close to critical
}

\author{
V.E.Sakhnyuk, A. V.Svidzinsky \\ Department of Theoretical and Mathematical Physics, \\ Lesya Ukrainka Volyn State University, \\ 13 Voli Avenue, 43000 Lutsk, Ukraine
}

Received October 30, 2002

\begin{abstract}
The behavior of the order parameter close to the superconductor-normal metal interface is researched. The thickness $d$ of the normal layer is arbitrary and the temperature is close to critical. The method of quasiorthogonality to asymptotics is used to obtain boundary conditions. The expression that describes the dependence of the current states on the normal layer's thickness is obtained. Asymptotic forms of solution at large and at small $d$ (relatively to the coherence length) are considered.
\end{abstract}

Key words: current states, the order parameter, the linear integral equation, asymptote

PACS: $74.50 .+r$

\section{Introduction}

There are numerous papers in which the problem of constructing the current states in an SNS junction ( $\mathrm{S}$ - superconductor, $\mathrm{N}$ - normal metal) is considered. However, the interest to the problem remains since some issues are still open or their description leads to complicated theoretical calculations. Naturally, the model approximations can be used here but they do not give an exact description of the phenomena under consideration.

Papers $[1,2]$ present the theory of the current states in an SNS junction in which the thickness of the normal layer $d \gg \xi_{0}$ and the temperature is close to critical. The presence of the electron reflection from the NS interface is taken into account in $[3,4]$. The case of a dirty superconductor has been investigated in [5]. The important moment which permits to execute the calculations to the very end is the condition $d \gg \xi_{0}$ being assumed. Taking into account this condition one can find corresponding asymptotics for the current.

*E-mail: sve@lab.univer.lutsk.ua 
There is no restriction on the thickness $d$ of the normal layer in this paper, i.e., the consideration also includes the microscopic junction when $d \sim \xi_{0}$. The theory is built for the temperature close to critical, since in this case one can use GinzburgLandau theory. The complete mesoscopic theory is enlisted only for calculating the parameter which determines the boundary condition.

\section{The behavior of the order parameter in an SNS junction close to NS interface}

Let the surfaces, that separate normal and superconductive metals, be planar and the axis $O Z$ be perpendicular to them. The superconductor occupies the region $|z|>d / 2$ while the normal metal occupies $|z|<d / 2$. The reflection of electrons from NS interface is absent, i.e. the transition coefficient $D=1$.

Near the NS interface, the superconductor is described by a linear integral equation for the order parameter $\Delta(z)$. The method of constructing this equation has been presented in [2]. The equation has the following form:

$$
\Delta(z)=\int_{-\infty}^{-d / 2} K\left(z-z^{\prime}\right) \Delta\left(z^{\prime}\right) \mathrm{d} z^{\prime}+\int_{d / 2}^{\infty} K\left(z-z^{\prime}\right) \Delta\left(z^{\prime}\right) \mathrm{d} z^{\prime} .
$$

Its kernel is

$$
K(z)=\frac{\pi \rho T_{\mathrm{c}}}{v_{0}} \sum_{\omega_{n}} \int_{0}^{1} \frac{\mathrm{d} x}{x} \exp \left(-\frac{2\left|\omega_{n}\right|}{v_{0} x}|z|\right),
$$

where $\omega_{n}=\pi T_{\mathrm{c}}(2 n+1)$ is the odd Matsubara frequency (for Fermi statistics); $\rho=|g| N(0)$ is dimensionless coupling constant, $N(0)$ is electron states density on the surface of the Fermisphere; $v_{0}$ is Fermi velocity. It is convenient to rewrite the equation (1) in such a manner that integration can be performed on the half-axis $z>0$. To this end, we shall introduce the even $\Delta_{s}(z)$ and the odd $\Delta_{a}(z)$ parts of the order parameter. Equations for them are as follows:

$$
\Delta_{s, a}(z)=\int_{d / 2}^{\infty}\left\{K\left(z-z^{\prime}\right) \pm K\left(z+z^{\prime}\right)\right\} \Delta_{s, a}\left(z^{\prime}\right) \mathrm{d} z^{\prime} .
$$

The sign plus corresponds to $\Delta_{s}(z)$, minus corresponds to $\Delta_{a}(z)$. Let us carry out the shift of variables $z$ and $z^{\prime}$ on $d / 2$ and denote the shifted functions by the same letters, i.e. $\Delta_{s, a}\{z+d / 2\} \rightarrow \Delta_{s, a}(z)$. Then, the integral equations take the following form:

$$
\Delta_{s, a}(z)=\int_{0}^{\infty}\left\{K\left(z-z^{\prime}\right) \pm K\left(z+z^{\prime}+d\right)\right\} \Delta_{s, a}\left(z^{\prime}\right) \mathrm{d} z^{\prime} .
$$

It is simpler to work with the equations which are written in dimensionless variables. Let us put $\zeta=z / \xi_{0}, a=d / \xi_{0}$, where $\xi_{0}=v_{0} / 2 \pi T_{\mathrm{c}}$ is the coherence 
length. Then,

$$
\begin{aligned}
\Delta_{s, a}(\zeta) & =\int_{0}^{\infty}\left\{K\left(\zeta-\zeta^{\prime}\right) \pm K\left(\zeta+\zeta^{\prime}+a\right)\right\} \Delta_{s, a}\left(\zeta^{\prime}\right) \mathrm{d} \zeta^{\prime} \\
K(\zeta) & =\frac{\rho}{2} \sum_{n} \int_{0}^{1} \frac{\mathrm{d} x}{x} \exp \left(-\frac{|2 n+1|}{x}|\zeta|\right) .
\end{aligned}
$$

One can show from (1) that the asymptotics of the order parameter on the infinity for both signs are linear, i.e.

$$
\begin{aligned}
& \Delta(\zeta) \stackrel{\text { as }}{=} \Delta_{+}^{\prime} \zeta+\Delta_{+}, \quad \zeta \rightarrow+\infty, \\
& \Delta(\zeta) \stackrel{\text { as }}{=} \Delta_{-}^{\prime} \zeta+\Delta_{-}, \quad \zeta \rightarrow-\infty .
\end{aligned}
$$

Under the infinity we mean the region $\zeta \gg \xi_{0}$. For both the even and the odd solutions we shall have, respectively:

$$
\Delta_{s}(\zeta) \stackrel{\text { as }}{=} C_{1}\left(\zeta+q_{1, \infty}\right), \quad \Delta_{a}(\zeta) \stackrel{\text { as }}{=} C_{2}\left(\zeta+q_{2, \infty}\right), \quad \zeta \rightarrow+\infty
$$

From (5) and (6) one can obtain the relations

$$
\begin{array}{ll}
C_{1}=\Delta_{+}^{\prime}-\Delta_{-}^{\prime}, & C_{1} q_{1, \infty}=\Delta_{+}+\Delta_{-}, \\
C_{2}=\Delta_{+}^{\prime}+\Delta_{-}^{\prime}, & C_{2} q_{2, \infty}=\Delta_{+}-\Delta_{-} .
\end{array}
$$

Excluding the constants $C_{1}$ and $C_{2}$ we get

$$
\begin{aligned}
& \left(\Delta_{+}^{\prime}-\Delta_{-}^{\prime}\right) q_{1, \infty}=\Delta_{+}+\Delta_{-}, \\
& \left(\Delta_{+}^{\prime}+\Delta_{-}^{\prime}\right) q_{2, \infty}=\Delta_{+}-\Delta_{-} .
\end{aligned}
$$

It is known $[1,2]$ that the constants $q_{1, \infty}$ and $q_{2, \infty}$ are not arbitrary. They are uniquely fixed by the solution of the linear integral equation. We have to calculate them, since they are involved in the expression for the density current.

There exist several methods which can be used for this purpose. In the paper [1] the variational method of Rits was used. The authors have built a functional minimization of which leads to the linear integral equation for the order parameter $\Delta(z)$. However, in the case of the junctions which have no left-right symmetry one cannot build an appropriate functional. To avoid this the Gal'orkin's method was used in the further studies. In the method it is unnecessary to know any functional. In the present work to calculate the required constants we use the so-called method of quasiorthogonality to asymptotics [5] which is simpler than the above mentioned.

The starting point is the equation (4), which may be considered only for the even part of the order parameter $\Delta_{s}(\zeta)$. Separating asymptotics on the infinity

$$
\Delta_{s}(\zeta)=C_{1}\left(\zeta+q_{1, \infty}+\psi(\zeta)\right)
$$


and substituting it into the equation (4) one obtains the following equation for a function $\psi(z)$

$$
\begin{aligned}
& \psi(\zeta)-\int_{0}^{\infty}\left\{K\left(\zeta-\zeta^{\prime}\right)+K\left(\zeta+\zeta^{\prime}+a\right)\right\} \psi\left(\zeta^{\prime}\right) \mathrm{d} \zeta^{\prime}= \\
& =\int_{0}^{\infty}\left\{K\left(\zeta+\zeta^{\prime}\right)+K\left(\zeta+\zeta^{\prime}+a\right)\right\} \zeta^{\prime} \mathrm{d} \zeta^{\prime} \\
& \quad-q_{1, \infty} \int_{0}^{\infty}\left\{K\left(\zeta+\zeta^{\prime}\right)-K\left(\zeta+\zeta^{\prime}+a\right)\right\} \mathrm{d} \zeta^{\prime} .
\end{aligned}
$$

Here we have used the formulas which are easy to check

$$
\begin{aligned}
\int_{0}^{\infty} K\left(\zeta-\zeta^{\prime}\right) \mathrm{d} \zeta^{\prime} & =1-\int_{0}^{\infty} K\left(\zeta+\zeta^{\prime}\right) \mathrm{d} \zeta^{\prime}, \\
\int_{0}^{\infty} K\left(\zeta-\zeta^{\prime}\right) \zeta^{\prime} \mathrm{d} \zeta^{\prime} & =\zeta+\int_{0}^{\infty} K\left(\zeta+\zeta^{\prime}\right) \zeta^{\prime} \mathrm{d} \zeta^{\prime} .
\end{aligned}
$$

Now, let us integrate the equation (8) by $\zeta$ from zero to infinity. Using the first formula of equation (9) we obtain a useful relation:

$$
\begin{aligned}
& \int_{0}^{\infty} \mathrm{d} \zeta \int_{0}^{\infty}\left\{K\left(\zeta+\zeta^{\prime}\right)-K\left(\zeta+\zeta^{\prime}+a\right)\right\} \psi\left(\zeta^{\prime}\right) \mathrm{d} \zeta^{\prime}= \\
& =\int_{0}^{\infty} \mathrm{d} \zeta \int_{0}^{\infty}\left\{K\left(\zeta+\zeta^{\prime}\right)+K\left(\zeta+\zeta^{\prime}+a\right)\right\} \zeta^{\prime} \mathrm{d} \zeta^{\prime} \\
& \quad-q_{1, \infty} \int_{0}^{\infty} \mathrm{d} \zeta \int_{0}^{\infty}\left\{K\left(\zeta+\zeta^{\prime}\right)-K\left(\zeta+\zeta^{\prime}+a\right)\right\} \mathrm{d} \zeta^{\prime} .
\end{aligned}
$$

The second important relation is obtained multiplying (8) by $\zeta$ and integrating by $\zeta$ from zero to infinity. Using the second formula of equation (9) one can find

$$
\begin{aligned}
& \int_{0}^{\infty} \mathrm{d} \zeta \zeta \int_{0}^{\infty}\left\{K\left(\zeta+\zeta^{\prime}\right)+K\left(\zeta+\zeta^{\prime}+a\right)\right\} \psi\left(\zeta^{\prime}\right) \mathrm{d} \zeta^{\prime}= \\
& =\int_{0}^{\infty} \mathrm{d} \zeta \zeta \int_{0}^{\infty}\left\{K\left(\zeta+\zeta^{\prime}\right)+K\left(\zeta+\zeta^{\prime}+a\right)\right\} \zeta^{\prime} \mathrm{d} \zeta^{\prime} \\
& \quad+q_{1, \infty} \int_{0}^{\infty} \mathrm{d} \zeta \int_{0}^{\infty}\left\{K\left(\zeta+\zeta^{\prime}\right)-K\left(\zeta+\zeta^{\prime}+a\right)\right\} \mathrm{d} \zeta^{\prime} .
\end{aligned}
$$

Let us introduce in (10) and (11) the function $q(\zeta)$ defined by a formula $q(\zeta)=$ $q_{1, \infty}+\psi(\zeta)$. Obviously, based on (6) one has $\lim _{\zeta \rightarrow \infty} q(\zeta)=q_{1, \infty}$. Substituting $\psi(\zeta)=$ 
$q(\zeta)-q_{1, \infty}$ into equations (10) and (11) one has the final form of the relations, that will be needed hereinafter:

$$
\begin{aligned}
\int_{0}^{\infty} \mathrm{d} \zeta \int_{0}^{\infty}\left\{K\left(\zeta+\zeta^{\prime}\right)-K\left(\zeta+\zeta^{\prime}+a\right)\right\} q\left(\zeta^{\prime}\right) \mathrm{d} \zeta^{\prime} & =I_{1}+I_{1}(a) \\
\int_{0}^{\infty} \mathrm{d} \zeta \zeta \int_{0}^{\infty}\left\{K\left(\zeta+\zeta^{\prime}\right)+K\left(\zeta+\zeta^{\prime}+a\right)\right\} q\left(\zeta^{\prime}\right) \mathrm{d} \zeta^{\prime} & =2 q_{1, \infty} I_{1}-I_{2}-I_{2}(a) .
\end{aligned}
$$

Here we have introduced such notations:

$$
\begin{aligned}
& I_{0}(a)=\int_{0}^{\infty} \mathrm{d} \zeta \int_{0}^{\infty} \mathrm{d} \zeta^{\prime} K\left(\zeta+\zeta^{\prime}+a\right), \quad I_{1}(a)=\int_{0}^{\infty} \mathrm{d} \zeta \int_{0}^{\infty} \mathrm{d} \zeta^{\prime} \zeta^{\prime} K\left(\zeta+\zeta^{\prime}+a\right), \\
& I_{2}(a)=\int_{0}^{\infty} \mathrm{d} \zeta \zeta \int_{0}^{\infty} \mathrm{d} \zeta^{\prime} \zeta^{\prime} K\left(\zeta+\zeta^{\prime}+a\right), \quad I_{k}=I_{k}(0), \quad k=0,1,2 .
\end{aligned}
$$

Let us clarify the sense of the above transformations. In the first case we multiplied the equation by unity, in the second one we multiplied by $\zeta$ and integrated by $\zeta$. Then, we did transformations to obtain zero in the left hand side of the equality. We did not obtain zero because functions 1 and $\zeta$ are not the solutions of the homogeneous equation. They are only its asymptotics for large $\zeta$. However, we neglected the terms, that are limited or increasing on the infinity. Relations (12) and (13) appear to be exact, and in [5] we called them the conditions of quasyorthogonality to asymptotics.

Let us take a trial function $\Gamma$ instead of $q(\zeta)$ in the relations (12) and (13). And let $\Gamma$ be an indefinite constant. We obtain

$$
\Gamma\left(I_{0}-I_{0}(a)\right)=I_{1}+I_{1}(a), \quad \Gamma\left(I_{1}+I_{1}(a)\right)=-I_{2}-I_{2}(a)+2 q_{1, \infty} I_{1} .
$$

From here one can find both $\Gamma$ and the expression for $q_{1, \infty}$

$$
q_{1, \infty}=\frac{1}{2 I_{1}}\left\{I_{2}+I_{2}(a)+\frac{\left(I_{1}+I_{1}(a)\right)^{2}}{I_{0}-I_{0}(a)}\right\} .
$$

The expression for $q_{2, \infty}$ differs from $q_{1, \infty}$ only in sign of $I_{k}(a)$, which is easy to understand from equation (4). Therefore,

$$
q_{2, \infty}=\frac{1}{2 I_{1}}\left\{I_{2}-I_{2}(a)+\frac{\left(I_{1}-I_{1}(a)\right)^{2}}{I_{0}+I_{0}(a)}\right\} .
$$

Certainly, formulas (14) and (15) give an approximate value of the coefficients $q_{1, \infty}$ and $q_{2, \infty}$ because we have taken the constant $\Gamma$ as a trial function instead of the exact solution for $q(\zeta)$. Let us note that the same results are obtained by other variation methods if the trial function is the same. However, the method of quasyorthogonality is the simplest. From (14) and (15) it also follows that $q_{1, \infty} \rightarrow \infty$, $q_{2, \infty} \rightarrow 0$ if $a \rightarrow 0$. 


\section{The dependence of the current density on the thickness of the normal layer in an SNS junction}

Let us calculate the current, which can flow through an SNS junction. The thickness $d$ of the normal layer is assumed to be arbitrary. An expression for the density of the current at a temperature close to critical in the Ginzburg-Landau theory $[1,6]$ is given by a formula:

$$
j(\zeta)=\mathrm{i} \frac{7 \zeta(3)}{16 \pi^{2}} \frac{e n v_{0}}{p_{0} \xi_{0} T_{\mathrm{c}}^{2}}\left(\Delta \frac{\mathrm{d} \Delta^{*}}{\mathrm{~d} \zeta}-\Delta^{*} \frac{\mathrm{d} \Delta}{\mathrm{d} \zeta}\right) .
$$

We shall calculate the density of the current in the region, where both the asymptotics of the solution of the linear integral equation on the infinity $\left(\zeta \gg \xi_{0}\right)$ and the asymptotics of the solution of the Ginzburg-Landau's equation for a small $\zeta$,

$$
\zeta \ll \xi(T), \quad \xi(T)=\sqrt{\frac{7 \zeta(3)}{12}} \frac{\xi_{0}}{\sqrt{1-T / T_{\mathrm{c}}}}
$$

overlap.

Therefore, substituting the expression for the order parameter into (16) we use its asymptotics form (5). It makes it possible to rewrite (16) in the form:

$$
j(\zeta)=\mathrm{i} \frac{7 \zeta(3)}{16 \pi^{2}} \frac{e n v_{0}}{p_{0} \xi_{0} T_{\mathrm{c}}^{2}}\left(\Delta_{+} \stackrel{*}{\prime}_{+}^{\prime}-\stackrel{*}{\Delta}_{+} \Delta_{+}^{\prime}\right)
$$

Of course, we could take the asymptotics on a minus infinity. Then, instead of the form $\Delta_{+} \stackrel{*}{\Delta}_{+}^{\prime}-\stackrel{*}{\Delta}_{+} \Delta_{+}^{\prime}$ we would have $\Delta_{-} \stackrel{*}{\Delta}_{-}^{\prime}-\stackrel{*}{\Delta}_{-} \Delta_{-}^{\prime}$. However, these both forms are equivalent. Really, the expressions $(7)$ can be written as follows:

$$
\left(\begin{array}{c}
\Delta_{+} \\
\Delta_{+}^{\prime}
\end{array}\right)=\hat{\mathcal{M}}\left(\begin{array}{c}
\Delta_{-} \\
\Delta_{-}^{\prime}
\end{array}\right), \quad \hat{\mathcal{M}}=\frac{1}{q_{1, \infty}-q_{2, \infty}}\left(\begin{array}{cc}
q_{1, \infty}+q_{2, \infty} & 2 q_{1, \infty} q_{2, \infty} \\
2 & q_{1, \infty}+q_{2, \infty}
\end{array}\right) .
$$

Expressing one of the forms by another we can see that they differ by a multiplier Det $\hat{\mathcal{M}}$ which is equal to unity. Therefore, we shall further neglect the argument in the density of the current. For further calculations it is convenient to exclude the derivatives of $\Delta$, using the expression (7). Thus, one obtains

$$
j=\mathrm{i} \frac{7 \zeta(3)}{32 \pi^{2}} \frac{e n v_{0}}{p_{0} \xi_{0} T_{\mathrm{c}}^{2}}\left(\frac{1}{q_{1, \infty}}-\frac{1}{q_{2, \infty}}\right)\left(\Delta_{+} \stackrel{*}{-}_{-}-\stackrel{*}{\Delta}_{+} \Delta_{-}\right) .
$$

Quantities $\Delta_{+}$and $\Delta_{-}$cannot be found from the solution of the linear integral equation because this solution is determined up to a multiplication constant. In order to determine this constant, we consider the condition of matching the solution of the linear integral equation with the solution of the Ginzburg-Landau equation. 
In the presence of the current states, the solution of the Ginzburg-Landau equation has the following form:

$$
\Delta(\zeta)=\exp \{ \pm \mathrm{i} \varphi / 2\} \Delta_{\infty} f(\zeta) \exp \{2 \mathrm{i} m \chi(\zeta)\}
$$

where

$$
f(\zeta)=\operatorname{th}\left(\frac{|\zeta|+\gamma-a / 2}{\sqrt{2} \xi(T)} \xi_{0}\right) .
$$

A phase of the order parameter is introduced here. It is bound up with a superfluid velocity: $\nabla \chi=v_{s}$. This function is considered to be continuous throughout. Specifically, we assume $\chi(a / 2)=\chi(-a / 2)=0$.

To describe the current states we should assume the presence of a phase difference between the banks of the junction. To this end, we introduce the phase factor $\exp \{ \pm \mathrm{i} \varphi / 2\}$, which is independent of the coordinate within each of the superconductors, but differs in both of them. We shall assume that the phase $\varphi / 2$ is for the right hand superconductor and the phase $-\varphi / 2$ is for the left hand one. The representation (20) makes it possible to write down the formula for the density current as follows:

$$
j=\frac{7 \zeta(3)}{16 \pi^{2}} \frac{e n v_{0} \Delta_{\infty}^{2}}{p_{0} \xi_{0} T_{\mathrm{c}}^{2}}\left(\frac{1}{q_{2, \infty}}-\frac{1}{q_{1, \infty}}\right) f_{+} f_{-} \sin \varphi
$$

where $f_{+}$and $f_{-}$are values of the function $f(\zeta)$ at $\zeta=a / 2$ and $\zeta=-a / 2$, respectively, i.e. $f_{+}=f_{-}=\operatorname{th}\left(\gamma \xi_{0} / \sqrt{2} \xi(T)\right)$.

Before obtaining a result for the current, let us consider the condition which follows from the relations (7). We write them down using the function $f(\zeta)$ which was introduced by the (20). After comparing both the real and the imaginary parts, the relation (7) takes the following form:

$$
\left\{\begin{array}{l}
2 q_{1, \infty} q_{2, \infty} f_{+}^{\prime}-\left(q_{1, \infty}+q_{2, \infty}\right) f_{+}+\left(q_{1, \infty}-q_{2, \infty}\right) f_{-} \cos \varphi=0 \\
4 m v_{s} \xi_{0} q_{1, \infty} q_{2, \infty} f_{+}+\left(q_{2, \infty}-q_{1, \infty}\right) f_{-} \sin \varphi=0 \\
2 q_{1, \infty} q_{2, \infty} f_{-}^{\prime}+\left(q_{1, \infty}+q_{2, \infty}\right) f_{-}+\left(q_{2, \infty}-q_{1, \infty}\right) f_{+} \cos \varphi=0 \\
4 m v_{s} \xi_{0} q_{1, \infty} q_{2, \infty} f_{-}+\left(q_{2, \infty}-q_{1, \infty}\right) f_{+} \sin \varphi=0
\end{array}\right.
$$

These equations have a solution that is not equal to zero, at the condition

$$
4 m v_{s} \xi_{0}=\left(\frac{1}{q_{2, \infty}}-\frac{1}{q_{1, \infty}}\right) \sin \varphi
$$

Taking into account the last relation, it is easy to find from (22) that

$$
f_{+}=f_{-}, \quad f_{+}^{\prime}=-f_{-}^{\prime}, \quad \frac{f_{+}^{\prime}}{f_{+}}=\frac{1}{2}\left(\frac{1}{q_{1, \infty}}+\frac{1}{q_{2, \infty}}\right)+\frac{1}{2}\left(\frac{1}{q_{1, \infty}}-\frac{1}{q_{2, \infty}}\right) \cos \varphi .
$$


Thus, we really have that $f_{+}=f_{-}$since the system is symmetrical, the relation $f_{+}^{\prime}=-f_{-}^{\prime}$ follows from the symmetry as well. To find the unknown constant $\gamma$, which is involved in the expressions for $f_{+}$and $f_{-}$, one uses the relations:

$$
\Delta_{+}^{\prime}=\frac{\Delta_{\infty} \xi_{0}}{\sqrt{2} \xi(T)} \mathrm{e}^{\mathrm{i} \varphi / 2} / \operatorname{ch} \frac{\gamma \xi_{0}}{\sqrt{2} \xi(\mathrm{T})}, \quad \Delta_{+}^{\prime} q_{\infty}=\Delta_{\infty} \operatorname{th} \frac{\gamma \xi_{0}}{\sqrt{2} \xi(T)} \mathrm{e}^{\mathrm{i} \varphi / 2}, \quad q_{\infty}=\frac{\Delta_{+}}{\Delta_{+}^{\prime}}
$$

These relations are obtained from the matching condition of the solution of the linear integral equation and the solution of the Ginzburg-Landau equation. We note that from (23) and (24) there follows an identity $q_{\infty}^{-1}=\cos ^{2}(\varphi / 2) / q_{1, \infty}+\sin ^{2}(\varphi / 2) / q_{2, \infty}$.

Based on (24) one has for $f_{+}$and $f_{-}$

$$
f_{+}=f_{-}=\operatorname{th}\left(\frac{\gamma \xi_{0}}{\sqrt{2} \xi(T)}\right)=\frac{q_{\infty} \xi_{0}}{\sqrt{2} \xi(T)}\left(\frac{1}{2}+{\sqrt{\frac{1}{4}+\frac{q_{\infty}^{2} \xi_{0}^{2}}{2 \xi^{2}(T)}}}^{-1} .\right.
$$

Thus, the expression for the density of the current can be written as follows:

$$
j=\frac{7 \zeta(3)}{16 \pi^{2}} \frac{e n v_{0} \Delta_{\infty}^{2}}{p_{0} \xi_{0} T_{\mathrm{c}}^{2}} \frac{q_{1, \infty}-q_{2, \infty}}{q_{1, \infty} q_{2, \infty}} \frac{q_{\infty}^{2} \xi_{0}^{2}}{2 \xi^{2}(T)} \sin \varphi\left(\frac{1}{2}+\sqrt{\frac{1}{4}+\frac{q_{\infty}^{2} \xi_{0}^{2}}{2 \xi^{2}(T)}}\right)^{-2}
$$

Taking into account the well-known relations:

$$
\xi^{2}(T)=\frac{7 \zeta(3)}{12} \frac{\xi_{0}^{2}}{1-T / T_{\mathrm{c}}}, \quad \Delta_{\infty}^{2}=\frac{8 \pi^{2} T_{\mathrm{c}}^{2}}{7 \zeta(3)}\left(1-T / T_{\mathrm{c}}\right)
$$

one can obtain a final expression for the density of the current in which there is no restriction on the thickness $d$ of the normal layer

$$
j=\frac{3}{7 \zeta(3)} \frac{e n v_{0}}{p_{0} \xi_{0}}\left(1-T / T_{\mathrm{c}}\right)^{2} \frac{q_{1, \infty}-q_{2, \infty}}{q_{1, \infty} q_{2, \infty}} \frac{q_{\infty}^{2} \sin \varphi}{\left(\frac{1}{2}+\sqrt{\frac{1}{4}+q_{\infty}^{2} \frac{6}{7 \zeta(3)}\left(1-T / T_{\mathrm{c}}\right)}\right)^{2}} .
$$

From the latter expression it is easy to obtain the asymptotics forms for the density current. At small $d,\left(d \ll \xi_{0}\right)$

$$
j=\frac{6}{7 \zeta(3)} \frac{e n v_{0}}{p_{0} \xi_{0}}\left(1-T / T_{\mathrm{c}}\right)^{2} q_{2, \infty} \frac{\cos (\varphi / 2)}{\sin ^{3}(\varphi / 2)} .
$$

At large $d,\left(d \gg \xi_{0}\right)$

$$
j=\frac{3}{7 \zeta(3)} \frac{e n v_{0}}{p_{0} \xi_{0}}\left(1-T / T_{\mathrm{c}}\right)^{2} \frac{q_{1, \infty}}{q_{2, \infty}}\left(q_{1, \infty}-q_{2, \infty}\right) \sin \varphi .
$$

The latter formula coincides with the result which was obtained in the paper [4], where from the very start it was assumed that $\left(d \gg \xi_{0}\right)$. 


\section{References}

1. Galaiko V.P., Svidzinsky A.V., Slyusarev V.A. // Zh. Eksp. Teor. Fiz., 1969, vol. 56, No. 3, p. 835-840.

2. Svidzinsky A.V. Space-inhomogeneous Problems of the Superconducting Theory. Moscow, Nauka, 1982.

3. Golubev L.V., Rakov Y.A., Svidzinsky A.V. // Physics of many partical systems, 1985, No. 7 , p. $40-45$.

4. Svidzinsky A.V., Golubev L.V. // Teor. Mat. Fiz., 1984, vol. 59, No. 1, p. 129-138 (in Russian).

5. Svidzinsky A.V., Sakhnyuk V.E. // Condens. Matter Phys., 2000, vol. 3, No. 3(23), p. $683-696$.

6. Svidzinsky A.V. The Microscopic Theory of Superconductivity. Lutsk, Vezha, 2001.

\section{Струмові стани в SNS-контакті при довільній товщині нормального прошарку для температур, близьких до критичної}

\section{В.Є.Сахнюк, А.В.Свідзинський}

Кафедра теоретичної і математичної фізики, Волинський державний університет ім. Лесі Українки, 43000 Луцьк, проспект Волі, 13

Отримано 30 жовтня 2002 р.

Досліджено поведінку параметра порядку поблизу границі розділу нормального металу та надпровідника. Товщина $d$ нормального металу вважається довільною, а температура близькою до критичної. Для одержання граничних умов використано метод квазіортогональності до асимптотики. Отримано залежність виразу для струму від товщини нормального прошарку, а також розглянуті його асимптотичні форми при великих і малих $d$ в порівнянні з довжиною когерентності $\xi_{0}$.

Ключові слова: струмові стани, параметр порядку, лінійне інтегральне рівняння, асимптотика

PACS: $74.50 .+r$ 
